\title{
Exact Solutions of Axially Symmetric Bianchi Type-I Cosmological Model in Lyra Geometry
}

\author{
A. Asgar ${ }^{1}$, M. Ansari ${ }^{2}$ \\ Department of Mathematics, North-Eastern Hill University, Permanent Campus, Shillong- 793022, Meghalaya, \\ India.
}

\begin{abstract}
In this paper we have obtained axially symmetric Bianchi type-I cosmological models for perfect fluid distribution in the context of Lyra's manifold. Exact solutions of the field equations are obtained by assuming the expansion $\theta$ in the model is proportional to the shear $\sigma$. This leads to the condition $A=B^{n}$ where $A$ and $B$ are scale factors and $n(\neq 0)$ is a constant. Some kinematical and physical parameters of the model have been discussed. The solutions are compatible with recent observations.
\end{abstract}

Keywords: Bianchi type models, Cosmology, Lyra geometry.

\section{Introduction}

Einstein proposed his general theory of relativity, in which gravitation is described in terms of geometry and it motivated the geometrization of other physical fields. One of the attempt in this direction was made by Weyl [1] who proposed a more general theory in which gravitation and electromagnetism is also described geometrically. But this theory was not accepted as it was based on non-integrability of length transfer. Later Lyra [2] introduced a gauge function i.e. displacement vector in Riemannian space-time which removes the non-integrability condition of a vector under parallel transport. This modified geometry was named as Lyra's geometry.

In continuation of investigations, Sen [3] and Sen and Dunn [4] proposed a new scalar-tensor theory of gravitation. They constructed an analog of the Einstein's field equations based on Lyra geometry.

Halford [5] pointed out that the constant displacement vector field $\phi_{i}$ in Lyra's geometry plays the role cosmological constant in the normal general relativistic treatment. It is shown by Halford [6] that the scalartensor treatment based on Lyra's geometry predicts the same effects, within observational limits as the Einstein's theory. Recently, several authors [7-10] studied cosmological models based on Lyra's geometry in various contexts. Sen and Vanstone [11], Bhamra [12], Karade and Borikar [13], Kalyanshetti and Waghmode [14], Reddy and Innaiah [15], Reddy and Venkateswarlu [16] have studied various cosmological models in Lyra's geometry with a constant displacement field. However, this restriction of the displacement field to be constant is merely one of convenience and there is no a priori reason for it. Soleng [17] has pointed out that the cosmologies based on Lyra's manifold with constant gauge vector $\phi$ will either include a creation field and be equal to Hoyle's creation field cosmology [18-20] or contain a special vacuum field, which together with the gauge vector term may be considered as a cosmological term. In the latter case the solutions are equal to the general relativistic cosmologies with a cosmological term. Beesham [21] considered FRW models with time dependent displacement field. He has shown that by assuming the energy density of the universe to be equal to its critical value, the models have $k=-1$ geometry.

Axially symmetric cosmological models have been studied in both Riemannian and Lyra geometries. In context of general relativity theory, by adapting the comoving coordinate system, these models with string dust cloud source are studied by Bhattacharaya and Karade [22]. They shown that some of these models are singular free even at an initial epoch. In the context of Lyra's geometry these models are studied in the presence of cosmic source and thick domain walls [23] and in the presence of perfect fluid distribution [24].

The purpose of this work is to analyse general features of axially symmetric Bianchi type-I cosmological models with time dependent displacement vector in the framework of Lyra geometry. This paper is organized as follows: In section II, we discuss the metric and field equations. In section III, we discuss solutions of the field equations. In section IV, we discuss some physical and kinematical parameters of the model. Finally, in section V, discussion and concluding remarks are given. 


\section{The Metric and Field equations}

We consider axially symmetric Bianchi type-I space -time

$$
d s^{2}=d t^{2}-A^{2} d x^{2}-B^{2}\left(d y^{2}+d z^{2}\right)
$$

where $A(t), B(t)$ are the cosmic scale factors.

The field equations in normal gauge for Lyra's manifold, as obtained by Sen [3] are

$$
R_{i}^{j}-\frac{1}{2} \operatorname{Rg}_{i}^{j}+\frac{3}{2} \phi_{i} \phi^{j}-\frac{3}{4} \phi_{k} \phi^{k} g_{i}^{j}=-\chi T_{i}^{j},
$$

where $\phi_{i}$ is the displacement vector field defined as $\phi_{i}=(0,0,0, \beta)$. Here $\beta=\beta(t), \chi=8 \pi G$ and other symbols have their usual meaning as in Riemannian geometry.

We take a perfect fluid form for the energy momentum tensor

$$
T_{i}^{j}=(p+\rho) u_{i} u^{j}-p g_{i}^{j},
$$

where $\rho$ and $p$ are the energy density and pressure of the cosmic fluid respectively together with comoving coordinates $u^{i} u_{i}=1$, where $u_{i}=(0,0,0,1)$.

The field Eq. (2) together with (3) for the metric (1) reduces to

$$
\begin{aligned}
& 2 \frac{\dot{A} \dot{B}}{A B}+\frac{\dot{B}^{2}}{B^{2}}-\frac{3}{4} \beta^{2}=\chi \rho, \\
& 2 \frac{\ddot{B}}{B}+\frac{\dot{B}^{2}}{B^{2}}+\frac{3}{4} \beta^{2}=-\chi p, \\
& \frac{\ddot{B}}{B}+\frac{\dot{A} \dot{B}}{A B}+\frac{\ddot{A}}{A}+\frac{3}{4} \beta^{2}=-\chi p .
\end{aligned}
$$

The energy conservation equation $T_{i ; j}^{j}=0$ leads to

and

$$
\dot{\rho}+(p+\rho)\left(\frac{\dot{A}}{A}+2 \frac{\dot{B}}{B}\right)=0
$$

Eq. (8) leads to

$$
\begin{gathered}
\frac{3}{2} \phi_{i}\left[\frac{\partial \phi^{j}}{\partial x^{j}}+\phi^{l} \Gamma^{j}{ }_{l j}\right]+\frac{3}{2} \phi^{j}\left[\frac{\partial \phi_{i}}{\partial x^{j}}-\phi_{l} \Gamma^{l}{ }_{i j}\right]-\frac{3}{4} g_{i}^{j} \phi_{k}\left[\frac{\partial \phi^{k}}{\partial x^{j}}+\phi^{l} \Gamma^{k}{ }_{l j}\right] \\
-\frac{3}{4} g_{i}^{j} \phi^{k}\left[\frac{\partial \phi_{k}}{\partial x^{j}}-\phi_{l} \Gamma^{l}{ }_{k j}\right]=0 .
\end{gathered}
$$

Eq. (9) is automatically satisfied for $i=1,2,3$.

For $i=4$, Eq. (9) leads to

$$
\begin{gathered}
\frac{3}{2} \beta\left[\frac{\partial}{\partial x^{4}}\left(g^{44} \phi_{4}\right)+\phi^{4} \Gamma^{4}{ }_{44}\right]+ \\
-\frac{3}{2} g^{44} \phi_{4}\left[\frac{\partial \phi_{4}}{\partial t}-\phi_{4} \Gamma^{4}{ }_{44}\right]-\frac{3}{4} g_{4}^{4} \phi_{4}\left[\frac{\partial \phi_{4}}{\partial x^{4}}+\phi^{4} \Gamma_{44}^{4}\right] \\
-\frac{3}{4} g_{4}^{4} g^{44} \phi_{4}\left[\frac{\partial \phi_{4}}{\partial t}-\phi^{4} \Gamma^{4}{ }_{44}\right]=0
\end{gathered}
$$

which leads to

$$
\frac{3}{2} \beta \dot{\beta}+\frac{3}{2} \beta^{2}\left(\frac{\dot{A}}{A}+2 \frac{\dot{B}}{B}\right)=0 .
$$

Here dot denotes differentiation with respect to cosmic time ' $\mathrm{t}$ '. 


\section{Solutions of the Field Equations}

Eqs. (4)-(6) are three equations in five unknowns viz. $A, B, \beta, p$ and $\rho$. In order to obtain explicit exact solutions, we assume that $\sigma \propto \theta$ which leads to

$$
A=B^{n} \text {, where } n(\neq 0) \text { is a constant. }
$$

and the equation of state is

$$
p=m \rho, \text { where } 0 \leq m \leq 1
$$

Now the set of Eqs. (4), (5), (6), (12) and (13) admit an exact solutions given by

$$
\begin{aligned}
& A=(D t+E)^{\frac{n}{n+2}}, \\
& B=(D t+E)^{\frac{1}{n+2}},
\end{aligned}
$$

where $D=\frac{k_{1}(n+1)}{(n-1)}, E=c(n+2)$ and $k_{1}$ and $c$ are constants of integration.

Thus the geometry of the axially symmetric Bianchi type-I cosmological model is described by the metric

$$
d s^{2}=d t^{2}-(D t+E)^{\frac{2 n}{n+2}} d x^{2}-(D t+E)^{\frac{2}{n+2}}\left(d y^{2}+d z^{2}\right) .
$$

\section{Some Physical and Kinematical Parameters of the Model}

From Eqs. (4), (5) and (13) we have

$$
\begin{gathered}
p=\frac{2 D(D-1) m(n+1)}{(n+2)^{2}(1-m) \chi(D t+E)^{2}}, \\
\rho=\frac{2 D(D-1)(n+1)}{(n+2)^{2}(1-m) \chi(D t+E)^{2}}, \\
\frac{3}{4} \beta^{2}=\frac{2 D^{2} n(1-m)+D^{2}(1-m)-2 D(D-1)(n+1)}{(n+2)^{2}(1-m)(D t+E)^{2}},
\end{gathered}
$$

where $m \neq 1$. At $t=0$, the model (16) becomes flat and the pressure $(p)$, energy density $(\rho)$ and gauge function $\beta$ have finite values.

Further, as time increases, the scale factors $A$ and $B$ increase indefinitely. The expansion scalar $(\theta)$ which determines the volume behaviour of the fluid given by

$$
\theta=\frac{D}{D t+E}
$$

At the initial epoch $t=0, \theta$ is finite and $\theta \rightarrow 0$ when $t \rightarrow \infty$. Hence there is finite expansion in the model.

Also, Hubble parameter $(H)$ is given by

$$
H=\frac{1}{3} \theta=\frac{1}{3}\left(\frac{D}{D t+E}\right)
$$

The volume element $(V)$ is given by

$$
V=S^{3}=A B^{2}=D t+E,
$$

where $S$ is the average scale factor. The equation (22) shows that the volume increases as the time increases, that is, the model (16) is expanding with time.

Shear scalar $(\sigma)$ is given by

$$
\sigma^{2}=\frac{1}{2}\left(\sum_{i=1}^{3} H_{i}^{2}-3 H^{2}\right)=\frac{D^{2}(n-1)^{2}}{3(n+2)^{2}(D t+E)^{2}},
$$

where $H_{1}=\frac{\dot{A}}{A}, H_{2}=H_{3}=\frac{\dot{B}}{B}$ are the directional Hubble's parameters in the directions of $\mathrm{x}, \mathrm{y}$ and $\mathrm{z}$ respectively. 
From Eqs. (20) and (23), we obtain

$$
\lim _{t \rightarrow \infty} \frac{\sigma^{2}}{\theta^{2}}=\frac{(n-1)^{2}}{3(n-2)^{2}}=\text { cons } \tan t .
$$

Therefore the model does not approach isotropy for large values of $\mathrm{t}$.

Deceleration parameter $(q)$ is given by

$$
q=2 .
$$

Also, anisotropy parameter $\left(A_{m}\right)$ for the model is obtained as

$$
A_{m}=\frac{2(n-1)^{2}}{(n+2)^{2}}=\text { cons } \tan t .
$$

Therefore the model has constant anisotropy parameter throughout the evolution of the universe. In this model particle horizon exists because

$$
\int_{t_{0}}^{t} \frac{d t}{V}=\int_{t_{0}}^{t} \frac{d t}{D t+E}=\left[\frac{1}{D} \log (D t+E)\right]_{t_{0}}^{t}
$$

is a convergent integral.

We have observed that at $t=-\frac{E}{D}$, the spatial volume vanishes and it increases with cosmic time. For $n=1$, the mean anisotropy parameter vanishes and the directional scale factor

$$
A(t)=B(t)=(D t+E)^{\frac{1}{3}} .
$$

Therefore, isotropy is achieved in the derived model for $n=1$. For this particular value of $n$, we observe that $A(t)=B(t)=S(t)$. Therefore the metric (1) reduces to flat FRW space-time. Thus, the derived model acquires flatness for $n=1$. But in the same spirit, the shear $(\sigma)$ vanishes for $n=1$. Hence we cannot choose $n=1$ in the derived model.

\section{Discussion and Concluding Remarks}

In the present study we have obtained exact solutions of Sen's equations in the presence of perfect fluid for axially symmetric Bianchi type-I cosmological models in normal gauge for Lyra's manifold. The expansion velocity $\dot{S}$ diverges as $t \rightarrow-\frac{E}{D}$. Hence the expansion of the universe is infinite as we approach the initial $t \rightarrow-\frac{E}{D}$. For the model (16) the above physical quantities like Hubble parameter $(H)$, expansion scalar $(\theta)$ and the shear scalar $(\sigma)$ are diverges as $t \rightarrow-\frac{E}{D}$. Thus the universe starts with an infinite rate of expansion and measure of anisotropy. This behaves like the big-bang model of the universe. The expansion ceases and the volume becomes infinitely large at large value of $t$ (i.e. $t \rightarrow \infty$ ). Hence the rate of expansion of the universe decreases with increase of time. Since deceleration parameter $q>0$, thus we find that the model (16) represents a decelerating universe. At $t \rightarrow \infty$, the pressure $p$ and energy density $\rho$ remain undetermined. All these physical quantities remain finite and physical significant at finite region. Since $\frac{\sigma^{2}}{\theta^{2}}=\operatorname{cons} \tan t$, therefore the model does not approach isotropy for large values of $t$. This model also has a point type singularity at $t=-\frac{E}{D}$ and it has constant anisotropy parameter $\left(A_{m}\right)$ throughout the evolution of the universe. For $n=1$, the derived model reduces to flat FRW space-time. It has a particle horizon. As $t \rightarrow \infty$, the shear dies out and the expansion stop. Thus the gauge function $\beta(t)$ is large in the beginning of the model but decays continuously during its evolution. Similar results can be obtained for Hoyle's creation field [18] if the creation field is time dependent. 


\section{References}

[1] H. Weyl, Sber. Preussische Akademie der Wissenschaften zu Berlin, 1918, 465

[2] G. Lyra, Über eine Modification der Riemannschen Geometrie, Mathematische Zeitschrift 54, 1951, 52-64.

[3] D. K. Sen, A static cosmological model, Zeitschrift fur Physik C 149, 1957, 311-323.

[4] D. K. Sen and K. A. Dunn, A scalar-tensor theory of gravitation in a modified Riemannian manifold, Journal of Mathematical Physics 12, 1971, 578-586.

[5] W. D. Halford, Cosmological theory based on Lyra's geometry, Australian Journal of Physics 23, 1970, 863-9.

[6] W. D. Halford, Scalar-tensor theory of gravitation in a Lyra manifold, Journal of Mathematical Physics 13, 1972, $1699-1703$.

[7] A. Pradhan, Cylindrically symmetric viscous fluid universe in Lyra geometry, Journal of Mathematical Physics 50, 2009, 022501-022513.

[8] S. Kumar and C. P. Singh, An exact Bianchi type-I cosmological models in Lyra's manifold, International Journal of Modern Physics A 23, 2008, 813-822.

[9] V. U. M. Rao, T. Vinutha and M. V. Santhi, Bianchi type-V cosmological model with perfect fluid using negative constant deceleration parameter in a scalar tensor theory based on Lyra manifold, Astrophysics Space Science 314, 2008, $213-216$.

[10] J. K. Singh, Exact solutions of some cosmological models in Lyra geometry, Astrophysics Space Science 314, 2008, 361 - 365.

[11] D. K. Sen and J. R. Vanstone, On Weyl and Lyra manifold, Journal of Mathematical Physics 13, 1972, $990-993$.

[12] K. S. Bhamra, A cosmological model of class one in Lyra's manifold, Australian Journal of Physics 27, 1974, 541-547.

[13] T. M. Karade and S. M. Borikar, Thermodynamic equilibrium of a gravitating sphere in Lyra's geometry, General Relativity and Gravitation 9, 1978, 431-436.

[14] S. B. Kalyanshetti and B. B. Waghmode, A static cosmological model in Einstein-Cartan theory, General Relativity and Gravitation 14, 1982, 823-830.

[15] D. R. K. Reddy and P. Innaiah, A plane symmetric cosmological model in Lyra manifold, Astrophysics Space Science 123, 1986, 49-52.

[16] D. R. K. Reddy and R. Venkateswarlu, A static conformally flat cosmological model in Lyra's manifold, Astrophysics Space Science 136, 1987, 183-186.

[17] H. H. Soleng, Cosmologies based on Lyra's geometry, General Relativity and Gravitation 19, 1987, 1213-1216.

[18] F. Hoyle, A new model for the expanding universe, Monthly Notices of the Royal Astronomical Society, 108, 1948, $372-382$.

[19] F. Hoyle and J. V. Narlikar, Time symmetric electrodynamics and the arrow of the time in cosmology. Proceedings of the Royal Society of London. Series A 277, 1964, 1-23.

[20] F. Hoyle and J. V. Narlikar, On the avoidance of singularities in C-field cosmology, Proceedings of the Royal Society of London Series A 278, 1964, 465-478.

[21] A. Beesham, FLRW cosmological models in Lyra's manifold with time dependent displacement field, Australian Journal of Physics 41, 1988, 833-842.

[22] S. Bhattacharya and T. M. Karade, Uniform anisotropic cosmological model with string source, Astrophysics Space Science 202, 1993, 69-75.

[23] D. R. K. Reddy and M. V. S. Rao, Axially symmetric cosmic strings and domain walls in Lyra geometry, Astrophysics Space Science 302, 2006, 157-160.

[24] V. U. M. Rao and T. Vinutha, Axially symmetric cosmological models in a scalar tensor theory based on Lyra manifold, Astrophysics Space Science 319, 2009, 161-167. 